\title{
Electronic properties of graphene/ZnO 2D-2D composite
}

\author{
R.M. Balabai ${ }^{1}$, A.V. Zdeshchyts ${ }^{2}$, D.V. Zalevskyi ${ }^{3}$ \\ Kryvyi Rih State Pedagogical University \\ 54, Gagarina Ave., 50085 Kryvyi Rih, Ukraine
}

E-mail:balabai@i.ual,bodehita@gmail.com²,zalev.denisk@mail.ru³

\begin{abstract}
Within the framework of methods of the electron density functional and the $a b$ initio pseudopotential, we have obtained the spatial distributions of the valence electrons density, the electron energy spectra and the Coulomb potential for model composite structures consisting of graphene or graphene oxide and $\mathrm{ZnO}$. They were computed using the above methods with the author's program complex. It has been determined that composite structures made of graphene and $\mathrm{ZnO}$ are more energy efficient to be formed with oxygen atomic plane of $\mathrm{ZnO}$ to graphene. Composite structures of graphene oxide and $\mathrm{ZnO}$ are more energy efficient as compared to composites on graphene.
\end{abstract}

Keywords: composites, graphene, graphene oxide, $\mathrm{ZnO}$, the electron density functional, $a b$ initio pseudopotential, electron structure.

doi: https://doi.org/10.15407/spqeo21.01.065

PACS 71.15.Mb, 73.22.-f

Manuscript received 04.01.17; revised version received 18.08.17; accepted for publication 24.01.18; published online 29.03.18.

\section{Introduction}

Heterojunctions are the main building units in up-to-date semiconductor devices, such as light-emitting diodes and solid-state lasers $[1,2]$. Since graphene and other twodimensional (2-D) materials were discovered [3-5], the so-called van der Waals heterojunctions (VDW) appeared, which offer many opportunities. As opposed to traditional heterojunctions, grown with molecular-beam epitaxy, VDW-heterojunctions do not require correspondence of crystal lattices at the interface, which allows almost unlimited material combinations. Among devices based on these heterojunctions, there are tunneling transistors [6], photodetectors [7], lightemitting diodes [8,9], and photoelectric devices [10]. Nowadays, the majority of VDW-heterostructures is produced by mechanical transmission of 2-D atomic layered crystals [5]. Although this method of laboratory testing can demonstrate physical concept of the device, it cannot be used in industry. Moreover, orientation of heterojunction is difficult to control precisely during connection of some materials $[11,14,15]$, and that can cause considerable variations of device characteristics [12]. Lately, VDW epitaxial growth of 2-D molecular crystal on graphene, which makes it possible to create organic/inorganic hybrid structures [13, 16, 17], has been shown. The epitaxial process has advantages of low temperature, atomic smooth and clean interface, precise control of morphology, and scaling possibilities $[13,16-21]$.
Up to date, much attention is drawn to composite structures of graphene (GR) or graphene oxide (GO), and some semiconductors, which show significant improvement in charge transmission, photovoltaic activity, thermal conductivity [22-25] regarding components of composite. In particular, the heterostructure $\mathrm{GO} / \mathrm{ZnO}$ is suitable for solar cells [26], transparent electric-diodes in optoelectronic devices, photocatalytic appliances, sensors [27-29], etc.

GO is an insulator, however, its electronic characteristics can be changed, when they are controlled, resulting in the degree of graphene oxidation. The parts of graphene with oxygen-bearing functional groups or molecules, added to the edge or middle of carbon lattice, are understood under graphene oxides. The range of these groups is vast: hydroxyl, phenol, carbonyl, carboxyl, aryl, ethereal, phosphoryl groups, etc. Graphene oxides, modified with polymers as polyethylene glycol, polyesters, polyvinyl, acrylate polymers and so on are their variations. One more oxide group consists of doping compound. Graphene oxides containing in their structure one or two atoms of boron, nitrogen, aluminum, phosphorus, silicium, sulfur or groups based on them, e.g. melamine, phosphine, silane, polysiloxane, sulfide etc., are well-known.

GO properties are distinguished by the character of chemical bond of oxygen (carboxyl, hydroxyl, or epoxide group) with carbon atoms. Meanwhile, oxygen atoms are accidentally distributed all over the graphene surface. It is a problem to receive information about GO structure 
directly [30]. The detailed research of these properties has been carried out recently at Cornell University, the USA [31], using the atomic-force microscope, the scanning transmission electron microscope, and the electron spectrometer of electron energy loss spectroscope.

Some researches of hybrid structures are focused on $\mathrm{ZnO}$-bearing photodetectors [32]. Nonstructural photodetectors, which were based on $\mathrm{ZnO}$, showed a relatively long period of response [33]. The recent accessible papers describe the attempts to create GR-ZnO nanocomposite, $\mathrm{ZnO}$ nanowires, GR-units/ $\mathrm{ZnO}$ films [34, 35], GR-ZnO nanorods [36, 37], and GR folded up in cavities of $\mathrm{ZnO}$ spheres [38]. Besides, in the research [39] it was reported about the resistance switching in GR-ZnO, which can be used as the resistive randomaccess memory. However, accounts on GO collection on $\mathrm{ZnO}$ films and application of $\mathrm{GO} / \mathrm{ZnO}$ heterostructures in UV-probing, the same as before, are very rare. In the investigation [40], the inexpensive, easy and large-scale method was used to cover zinc oxide films with GO nanoplates. They used thin $\mathrm{ZnO}$ films as templates for GO to increase efficiency in distribution of photogenerated electron-hole pairs, which are created during the UV-radiation. These hybrid heterostructures showed reproducible, fast, and uniform response on UV illumination as compared to single $\mathrm{ZnO}$ films, because of high transport properties of carbon nanostructures.

For extension of information about electronic properties of composite structures of graphene or graphene oxide and $\mathrm{ZnO}$, in this article they are calculated using such methods as electron density functional and first-principles pseudopotential based on proper program code [41]. There determined were the spatial distributions of the valence electrons density, width of the band gap, Coulomb potentials along the chosen directions.

\section{Objects and methods of research}

The basic states of the electron-nucleus systems were detected by means of the self-consistent solution of the Kohn-Sham equations. Electronic variables were only determined with the atomic cores fixed. Following Kohn-Sham [18, 19], the electronic density was written down in terms of occupied orthonormal one-particle wave functions:

$$
n(\mathbf{r})=\sum_{i}\left|\psi_{i}(\mathbf{r})\right|^{2}
$$

The point on the surface of potential energy in the Born-Oppenheimer approximation was determined as a minimum energy functional with regard to the wave functions:

$$
\begin{aligned}
& E\left[\left\{\psi_{i}\right\},\left\{R_{j}\right\},\left\{\alpha_{v}\right\}\right]= \\
& \sum_{i} \int_{\Omega} d^{3} r \psi_{i}^{*}(\mathbf{r})\left[-\frac{\hbar^{2}}{2 m} \nabla^{2}\right] \psi_{i}(\mathbf{r})+U\left[\{n(\mathbf{r})\},\left\{R_{j}\right\}\left\{\alpha_{v}\right\}\right],
\end{aligned}
$$

where $\left\{R_{j}\right\}$ are coordinates of atomic cores, $\left\{\alpha_{v}\right\}-$ any external influences on the system.
In the generally accepted formulation, minimization of the energy functional (2) with respect to one-particle orbitals with additional orthonormal constraint on the one-particle orbitals $\psi_{i}(\vec{r})$ results in Kohn-Sham oneparticle equations [20]:

$\left\{-\frac{\hbar^{2}}{2 m} \nabla^{2}+\frac{\partial U}{\partial n(\mathbf{r})}\right\} \psi_{i}(\mathbf{r})=\varepsilon_{i} \psi_{i}(\mathbf{r})$.

In the solution of these equations, the pseudopotential formalism was used, according to which a solid is considered as a set of valence electrons and the ion cores. In the pseudopotential approximation, the operator of pseudopotential $V_{P S}$, which describes interaction of valence electrons with the core, is small, and the corresponding pseudo-wavefunction is smooth. The pseudopotential is required to correctly represent the long range interactions of the core and to produce pseudowavefunction solutions that approach the full wavefunction outside a core radius $r_{c}$. In addition, it is desired that the pseudopotential is transferable, which means that one and the same pseudopotential can be used in calculations of different chemical environment resulting in calculations with comparable accuracy. For example, Bachelet, Hamann and Schliiter proposed an analytic fit to the pseudopotentials. This ab initio pseudopotential is used by us.

The total crystalline potential is constructed as the sum of ion pseudopotentials that are not overlapping and associated with ions (nucleus + core electrons), located at the $\vec{R}_{S}$ positions that are periodically repeated for crystals:

$V_{\text {cryst }}(\vec{r}) \rightarrow V_{P S}(\vec{r})=\sum_{\vec{p}} \sum_{S} \hat{V}_{S}^{P S}\left(\vec{r}-\vec{p}-\vec{R}_{S}\right)$.

For nonperiodic systems, such as a thin film or a cluster, the problem of lack of periodicity is circumvented by use of the supercell method. Namely, the cluster is periodically repeated, but the distance between each cluster and its periodic images are so large that their interaction is negligible. The ubiquitous periodicity of the crystal (or artificial) lattice produces a periodic potential and thus imposes the same periodicity on the density (implying Bloch's Theorem). The KohnSham potential of a periodic system exhibits the same periodicity as the direct lattice and the Kohn-Sham orbitals can be written in the Bloch form:

$$
\psi(\vec{r})=\psi_{i}(\vec{r}, \vec{k})=\exp (i \vec{k} \cdot \vec{r}) u_{i}(\vec{r}, \vec{k})
$$

where $\vec{k}$ is a vector in the first Brillouin zone. The functions $u_{i}(\vec{r}, \vec{k})$ have the periodicity of the direct lattice. The index $i$ runs over all states. The periodic functions $u_{i}(\vec{r}, \vec{k})$ are expanded in the plane wave basis. This heavily suggests to use plane waves as the generic basis set in order to expand the periodic part of the orbitals. Since these plane waves form a complete and orthonormal set of functions, they can be used to expand orbitals according to: 

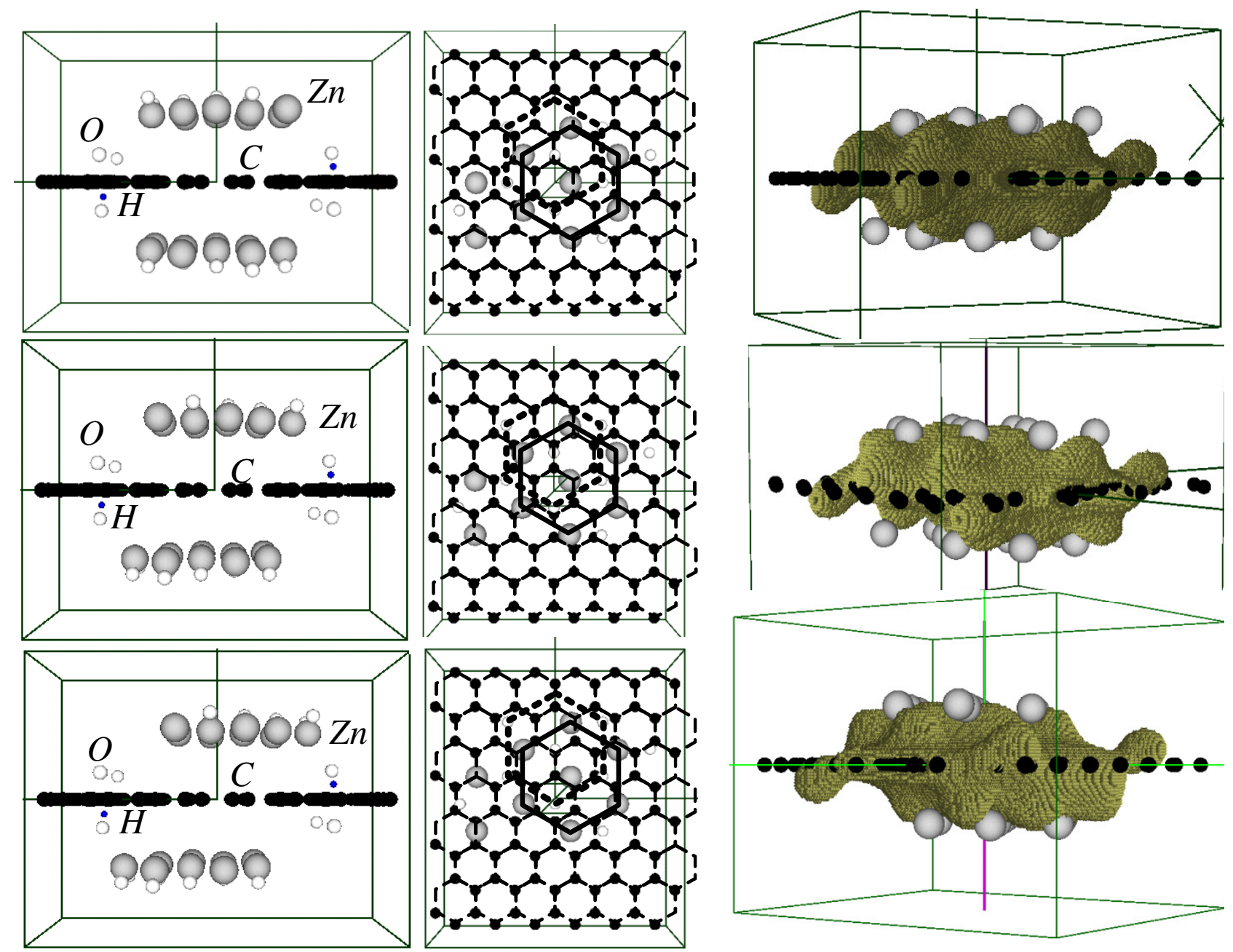

Fig. 1. The stage of $\mathrm{ZnO}$ bilayer shift on the graphene plate along the whole Y-axis. Top to bottom: initial position, shift by $0.65 \AA$, $1.3 \AA$ A. Left to right: lateral view of the cell, plan view and the view with electron density in the interval of iso-dimensions $0.5 \ldots 0.4$ from maximum in object 3 .

$$
\Psi_{j}(\vec{k}, \vec{r})=\frac{1}{\sqrt{N_{0}} \sqrt{\Omega}} \sum_{\vec{G}} b_{J}(\vec{k}+\vec{G}) \exp (i(\vec{k}+\vec{G}) \vec{r})
$$

where $\vec{G}$ is the vector in the reciprocal space, $\Omega$ is the volume of the elemental cells, which consists of a periodic crystal or an artificial super-lattice, when reproducing nonperiodic objects.

The equation (3) after the Fourier transform to the reciprocal space has the form:

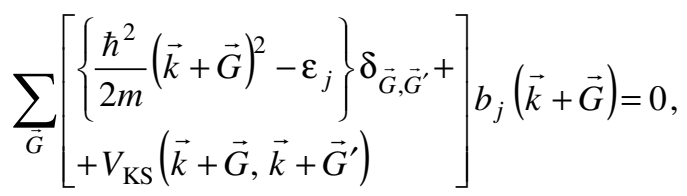

where $V_{\mathrm{KS}}$ is the Kohn-Sham potential:

$$
\begin{aligned}
& V_{\mathrm{KS}}\left(\vec{k}+\vec{G}, \vec{k}+\vec{G}^{\prime}\right)= \\
& V_{p s}\left(\vec{k}+\vec{G}, \vec{k}+\vec{G}^{\prime}\right)+V_{H}\left(\vec{G}^{\prime}-\vec{G}\right)+V_{x c}\left(\vec{G}^{\prime}-\vec{G}\right),
\end{aligned}
$$

where $V_{x c}$ is the exchange and correlation potential. To calculate it, we used Ceperley-Alder's approximation that has been parameterized by Perdew and Zunger.
In the general case, the expressions describing the potentials of interactions are complex. The use of the atomic bases containing the inversion operation in the point symmetry group leads to the fact that the Fouriercomponents in the expansion of all expressions are real.

The main value in the formalism of the functional of the electron density is the charge density. It is estimated from a self-consistent solution of equations (6), which should be performed in all points of the nonreduced section of the Brillouin zone:

$$
\rho(\vec{G})=\frac{2}{N_{T}} \sum_{\vec{k}} \sum_{\vec{j}} \sum_{\alpha \in T} \sum_{\vec{G}^{\prime}} b_{j}^{*}\left(\vec{k}+\vec{G}^{\prime}+\alpha \vec{G}\right) b_{j}\left(\vec{k}+\vec{G}^{\prime}\right) \text {. }
$$

where the index $j$ runs over all occupied states, $\vec{k}$ is a vector in the first Brillouin zone, $N_{T}$ is the number of the operators $\alpha$ in the point group $T$ of the atomic basis, and the factor 2 takes into account the spin degeneracy.

The estimated effort can be reduced, if there is the integral over the Brillouin zone to approximate by summing over special points of the Brillouin zone. It is possible to replace with satisfactory precision the summation by the finite number of special points to one point in the Brillouin zone. It is possible to restrict only 
the $\Gamma$-point in the Brillouin zone, especially as it relates to the artificial periodic systems.

Distribution of electrons along the energy zones for $\Gamma$-state of the investigated structures was found by means of numerical calculation of derivative $\lim _{\Delta E \rightarrow 0} \Delta N / \Delta E$ (where $\Delta N$ is a number of the allowed states for the $\Delta E$ interval of energy). The one-particle energy spectrum was obtained from calculation of the eigenvalues of the Kohn-Sham matrix. In accordance with ideology of the electronic density functional, the occupied states at absolute zero temperature were determined. It allowed to determine position of the last occupied state, their number being half the number of electrons (due to ignoring the spin of the electron), and position of the first free states.

The Coulomb potential along the given direction was calculated using the formula that in the reciprocal space has the form:

$$
V_{h}(\vec{G})=\frac{4 \pi e^{2} \rho(\vec{G})}{G^{2}},
$$

where $\rho(\vec{G})$ is the Fourier component of the electron density (8).

Developing the GO atomic model, the following rules were considered. First, the GO consisted of the graphene layer, the hydroxyl groups, and oxygen atoms related via the single bond with two adjacent atoms of graphene. Second, the equal amount of the functional groups was chosen on each side of the graphene plate. Third, pairs of the functional groups could not be added to the atom of carbon.

The composite patterns of graphene (or graphene oxide) and $\mathrm{ZnO}$ were designed by superimposing fragments of the bilayer $\mathrm{ZnO}$ film on two sides of a fragment of graphene or the graphene oxide plates. The bilayer $\mathrm{ZnO}$ films with wurtzite structure were oriented to the graphene either its atomic oxygen plane or its $\mathrm{Zn}$ atomic one.

The possibility for bilayer $\mathrm{ZnO}$ to have a wurtzite structure is proved in the works $[42,43]$. Tusche et al. [43] revealed two monolayer of $\mathrm{ZnO}$ grown on $\mathrm{Ag}(111)$ substrate by using surface X-ray diffraction and scanning tunneling microscopy. They also showed that transition to the bulk wz-ZnO structure occurs in the 3-4 monolayer coating. The measured $\mathrm{Zn}-\mathrm{O}$ bond length of the planar hexagonal structure $1.93 \AA$ is slightly larger than the value of $1.895 \AA$ calculated for the bond length of the monolayer of $\mathrm{ZnO}$. This situation implies that the effect of the $\operatorname{Ag}(111)$ substrate may be negligible. The authors of [42] investigated the atomic structure and stability of bilayer $\mathrm{ZnO}$ honeycomb structure. Their ab initio molecular dynamics calculations at $750 \mathrm{~K}$ indicate that the bilayer remains stable at $750 \mathrm{~K}$ after $3.0 \mathrm{ps}$.

In our calculations, the approach of artificial superlattice constructing was used. The artificial translational symmetry was inserted by building the super-lattice with the primitive cell of tetragonal type with atomic base containing all information about the investigated system.

The primitive cell of the super-lattice with dimensions $a=14.76 \AA, b=17.04 \AA$ in XY-plane and atomic bases were developed for reproduction of finite graphene or oxide graphene plates covered with finite fragments of $\mathrm{ZnO}$ film in XY-plane, these are shown in Fig. 1. The size of primitive cell in direction $\mathrm{Z}$ was chosen to avoid interaction of transmitting structures. The calculation was made only for $\Gamma$-point of Brillouin zone of the super-lattice. In calculations, these objects were distinguished:

object 1: graphene plate covered with fragments of $\mathrm{ZnO}$ bilayer that are oriented towards graphene with $\mathrm{O}$ plane, symbolic notation $(\mathrm{ZnO}+\mathrm{G})$, the size of cell towards Z-direction was $c=10.58 \AA$; the base consisted of $18 \mathrm{Zn}$ atoms, $18 \mathrm{O}$ atoms, and $96 \mathrm{C}$ atoms;

object 2: graphene plate covered with fragments of $\mathrm{ZnO}$ bilayer oriented towards graphene with Zn-plates; symbolic notation $(\mathrm{OZn}+\mathrm{G})$, the size of cell towards $\mathrm{Z}$ direction was $c=10.58 \AA$, the base consisted of $18 \mathrm{Zn}$ atoms, $18 \mathrm{O}$ atoms, and $96 \mathrm{C}$ atoms;

object 3: graphene oxide plate covered with fragments of $\mathrm{ZnO}$ bilayer oriented towards the oxide with O-plane, symbolic notation $(\mathrm{ZnO}+\mathrm{GO})$, the size of cell towards Z-direction was $c=13.23 \AA$, the base consisted of $18 \mathrm{Zn}$ atoms, $24 \mathrm{O}, 96 \mathrm{C}$, and $2 \mathrm{H}$ atoms;

object 4: graphene oxide plate covered with fragments of $\mathrm{ZnO}$ bilayer that was oriented towards the oxide with $\mathrm{Zn}$-plane; symbolic notation $(\mathrm{OZn}+\mathrm{GO})$, the size of cell towards Z-direction was $13.23 \AA$, the base consisted of $18 \mathrm{Zn}$ atoms, $24 \mathrm{O}, 96 \mathrm{C}$, and $2 \mathrm{H}$ atoms.

To check the compliance of crystal lattices and orientation of the components on the boundary of the distribution of composite structures on their electric properties, the fragments of $\mathrm{ZnO}$ films was shifted relatively to the graphene layer or its oxide (Fig. 1). The distance between $\mathrm{ZnO}$ bilayer and graphene or the highest atoms of its oxide corresponded to the distances between bilayers in the crystal $\mathrm{ZnO}$. The electron density of the analyzed atomic systems coordinated for some periods of iteration, the atomic base was not optimized.

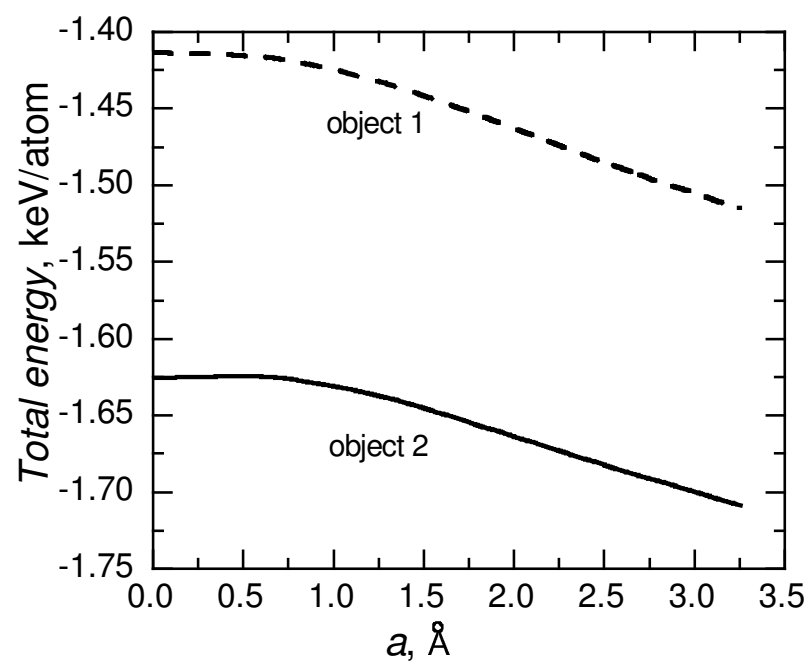

Fig. 2. Energy profiles of shift of fragments of $\mathrm{ZnO}$ bilayers for different orientation along the graphene plates. 


\section{Results of calculation and their discussion}

The analysis of Coulomb potential change computed in different positions of composites gives us an opportunity to assert that its biggest amount is formed under $\mathrm{ZnO}$ film and almost does not depend on where it is organized: on graphene or its oxide.

Figs 2 and 3 describe energy profiles of shift of fragments of $\mathrm{ZnO}$ bilayers with different orientation along graphene or its oxide plates. It is shown that the object 1 that is made of graphene plate covered with fragments of $\mathrm{ZnO}$ bilayers oriented towards graphene with its O-plane has the total energy, the value of which is less than that of the object 2, where $\mathrm{ZnO}$ is oriented towards graphene with its Zn-plane. This energy decrease in atomic system of the object 1 is related with the reaction of $\mathrm{ZnO}$ layer and graphene, which is more intensive when O-plane is oriented towards graphene. Spatial distributions of the valence electrons density (depicted in Fig. 4) prove the presence of this reaction, they have electron interchange zones between graphene and $\mathrm{ZnO}$, which are pronounced clearer for the object 1 . The shift of $\mathrm{ZnO}$ bilayer along the graphene surface changes a bit its total energy for both objects 1 and 2 .

The shift of fragments of $\mathrm{ZnO}$ bilayers on graphene oxide happens with lower energy than shift on graphene - almost half as much (see Fig. 3). This decrease in energy sum of atomic system occurs because of more intensive electron interaction in it, which is proved by electron density distribution in Fig. 1, the right column. Besides, the energy profile following $\mathrm{ZnO}$ shift on graphene oxide has a nonmonotonic character, which is clearer pronounced in the case, when graphene is close to atomic Zn-plane. Nonmonotonicity is related with random distribution of oxygen-bearing groups on graphene surface.

As for leading properties of the analyzed structures, they were determined from the analysis of band gap value. The band gap value is sensitive to the orientation of $\mathrm{ZnO}$ layer relatively to the graphene plane as well as to graphene oxide: it disappears in some cases of disposition of the components (Figs. 5 and 6).

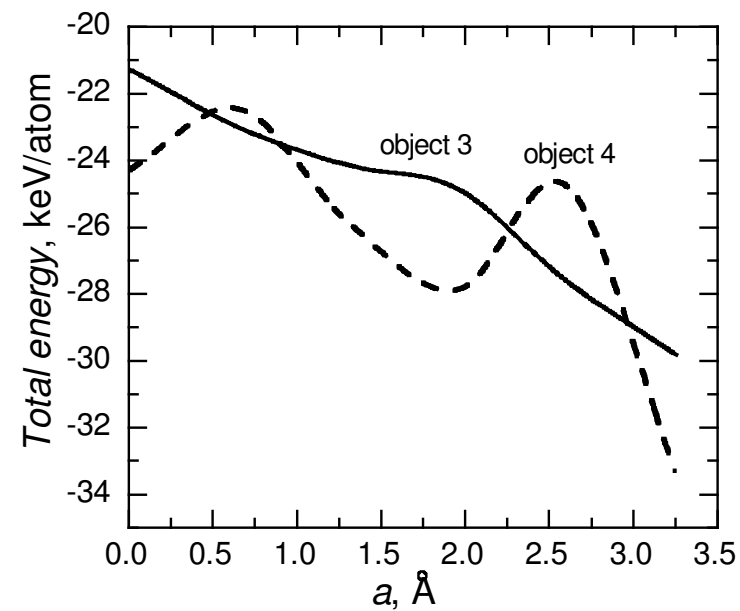

Fig. 3. Energy profiles of shift of fragments of $\mathrm{ZnO}$ bilayers for different orientation along the graphene oxide plate.

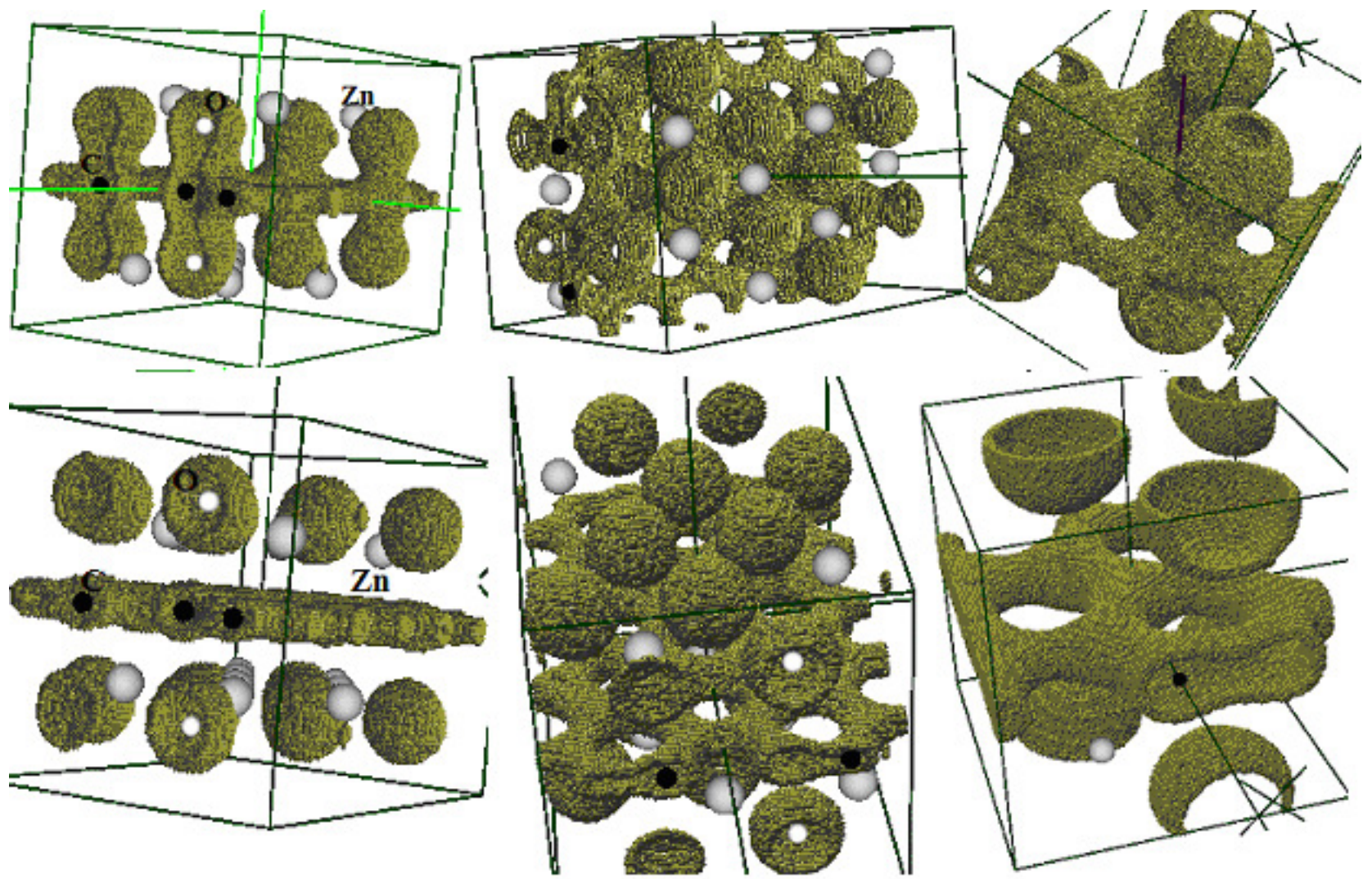

Fig. 4. Spatial density distributions of valence electrons for Zn-O/GR/O-Zn, object 1 (above), for heterostructures- O-Zn /GR/Zn-O, object 2 (below). 


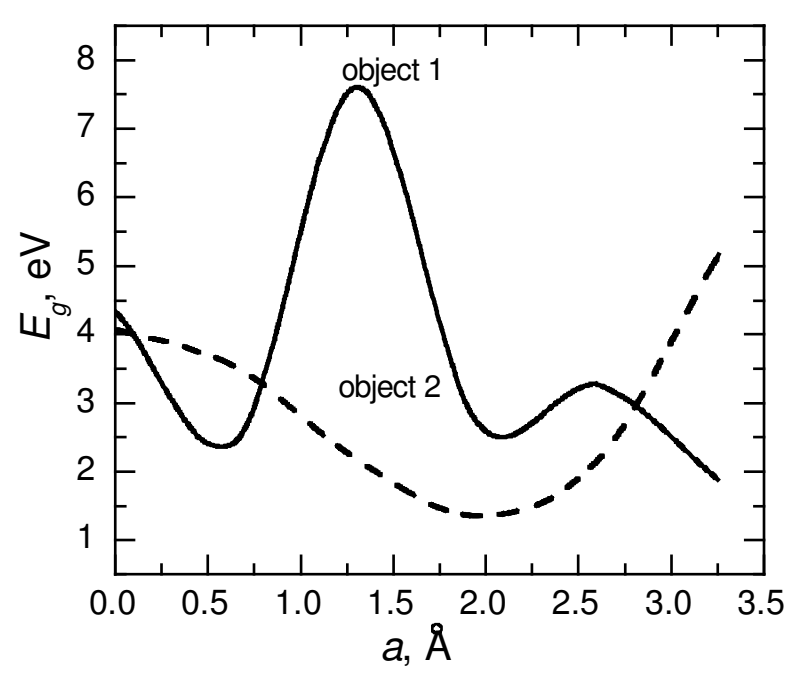

Fig. 5. Dependence of the band gap value on $\mathrm{ZnO}$ fragment shift along the graphene plate.

\section{Conclusions}

Spatial distribution of valence electrons, density of electron states, the energy band gap, and Coulomb potentials along the set directions for model composite structures consisting of graphene or graphene oxide and $\mathrm{ZnO}$ were computed using the methods of electron density functional theory and first-principles pseudopotentials being based on the authors' program complex.

It has been ascertained that the highest Coulomb potential value is formed under $\mathrm{ZnO}$ film and does not practically depend on where composite is organized: on graphene or graphene oxide.

It has been shown that the band gap value is sensitive to the orientation of $\mathrm{ZnO}$ relatively to the graphene plane as well as to graphene oxide: it disappears in some cases of disposition of the components.

It has been ascertained that the composite structures made of graphene and $\mathrm{ZnO}$ are more energy efficient to be formed with oxygen atomic plane of $\mathrm{ZnO}$ to graphene. Composite structures of graphene oxide and $\mathrm{ZnO}$ are more energy efficient as compared to composites on graphene.

\section{References}

1. Kroemer H. Heterostructure bipolar transistors and integrated circuits. Proc. IEEE. 1982. 70, No 13.

2. Sze S.M. and Ng K.K. Physics of Semiconductor Devices. Wiley, Hoboken, NJ, USA, 2006.

3. Novoselov K.S. and Geim A.K. The rise of grapheme. Nature Mater. 2007. 6. P. 183-191.

4. Wang Q., Kalantar-Zadeh K., Kis A., Coleman J.N., and Strano M.S. Electronics and optoelectronics of two-dimensional transition metal dichalcogenides. Nat. Nanotechnol. 7. 2012. P. 699-712.

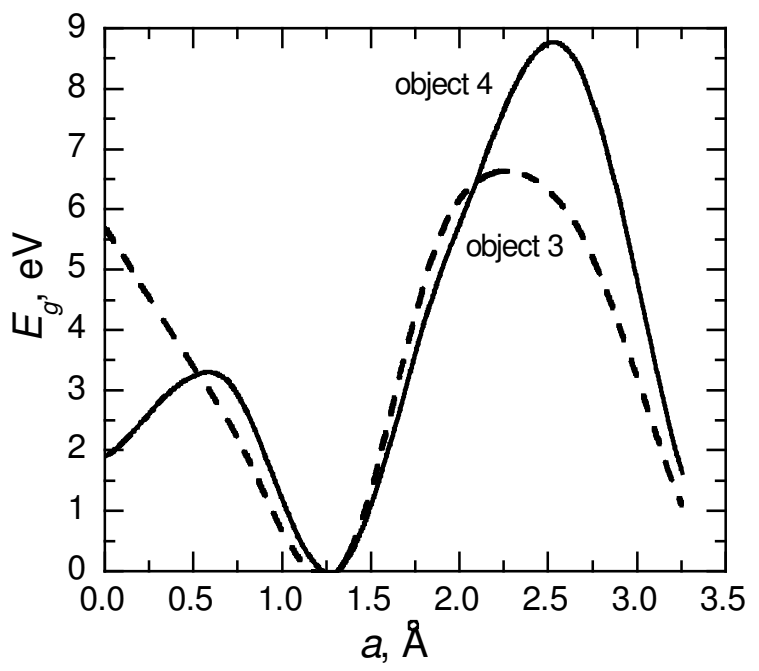

Fig. 6. Dependence of the band gap value on $\mathrm{ZnO}$ fragment shift along the graphene oxide plate.

5. Geim A.K. and Grigorieva I.V., Van der Waals heterostructures. Nature. 2013. 499. P. 419-425.

6. Britnell L., Gorbachev R.V., Jalil R. et al. Fieldeffect tunneling transistor based on vertical graphene heterostructures. Science. 2012. 335. P. 947-950.

7. Yu W.J., Liu Y., Zhou H., Yin A., Li Z., Huang Y., and Duan X. Highly efficient gate-tunable photocurrent generation in vertical heterostructures of layered materials. Nat. Nanotechnol. 2013. 8. P. 952-958.

8. Ross J.S., Klement P., Jones A.M., Ghimire N.J., Yan J., Mandrus D.G., Taniguchi T., Watanabe K., Kitamura K., Yao W., Cobden D.H., and Xu X. Electrically tunable excitonic light-emitting diodes based on monolayer $\mathrm{WSe}_{2} p-n$ junctions. Nat. Nanotechnol. 2014. 9. P. 268-272.

9. Withers F., Del Pozo-Zamudio O., Mishchenko A. et al. Light-emitting diodes by band-structure engineering in van der Waals heterostructures. Nature Mater. 2015. 14. P. 301-306.

10. Furchi M.M., Pospischil A., Libisch F., Burgdorfer J., and Mueller T. Photovoltaic effect in an electrically tunable van der Waals heterojunction. Nano Lett. 2014. 14, No 8. P. 4785-4791.

11. Yang W., Chen G., Shi Z. et al. Epitaxial growth of single-domain graphene on hexagonal boron nitride. Nat. Mater. 2013. 12. P. 792-797.

12. Fallahazad B., Lee K., S. Kang et al. Gate-tunable resonant tunneling in double bilayer graphene heterostructures. Nano Lett. 2015. 15, No 1. P. 428433.

13. He D., Zhang Y., Wu Q. et al. Two-dimensional quasi-freestanding molecular crystals for highperformance organic field-effect transistors. Nat. Commun. 2014. 5. P. 5162.

14. Kory M.J., Worle M., Weber T., Payamyar P., van de Poll S.W., Dshemuchadse J., Trapp N., and Schluter A.D. Gram-scale synthesis of two- 
dimensional polymer crystals and their structure analysis by X-ray diffraction. Nat. Chem. 2014. 6. P. 779-784.

15. Kissel P., Murray D.J., Wulftange W.J., Catalano V.J., and King B.T. A nanoporous two-dimensional polymer by single-crystal-to-single-crystal photopolymerization. Nat. Chem. 2014. 6. P. 774-778.

16. Kim K., Lee T.H., Santos E.J.G., Jo P.S., Salleo A., Nishi Y., and Bao Z. Structural and electrical investigation of C60-graphene vertical heterostructures. ACS Nano. 2015. 9. P. 5922-5928.

17. Kim K., Santos E.J.G., Lee T.H., Nishi Y., and Bao Z. Epitaxially grown strained pentacene thin film on graphene membrane. Small. 2015. 11, No 17. P. 2037.

18. Dean C.R., Young A.F., Meric I. et al. Boron nitride substrates for high-quality graphene electronics. Nat. Nanotechnol. 2010. 5. P. 722-726.

19. Lee C., Schiros T., Santos E.J.G. et al. Epitaxial growth of molecular crystals on van der Waals substrates for high-performance organic electronics. Adv. Mater. 2014. 26. P. 2812-2817.

20. Hlaing H., Kim C., Carta F., Nam C., Barton R.A., Petrone N., Hone J., and Kymissis I. Low-voltage organic electronics based on a gate-tunable injection barrier in vertical graphene-organic semiconductor heterostructures. Nano Lett. 2015. 15. P. 69-74.

21. Parui S., Pietrobon L., Ciudad D., Velez S., Sun X., Casanova F., Stoliar P., and Hueso L.E. Gatecontrolled energy barrier at a graphene/molecular semiconductor junction. Adv. Funct. Mater. 2015. 25. P. 2972-2979.

22. Liu W., Cai J., Li Z. Self-assembly of semiconductor nanoparticles/reduced graphene oxide (RGO) composite aerogels for enhanced photocatalytic performance and facile recycling in aqueous photocatalysis. ACS Sustainable Chem. Eng. 2015. 3, No 2. P. 277-282.

23. Gao N., Fang X. Synthesis and development of graphene-inorganic semiconductor nanocomposites. Chem. Rev. 2015. 115, No 16. P. 8294-8343.

24. Yang M.-Q., Zhang N., Pagliaro M., Xu Y.-J. Artificial photosynthesis over graphene-semiconductor composites. Are we getting better? Chem. Soc. Rev. 2014. 43. P. 8240-8254.

25. Xing M., Shen F., Qiu B., Zhang J. Highly dispersed boron doped graphene nanosheets loaded with $\mathrm{TiO}_{2}$ nanoparticles for enhancing $\mathrm{CO}_{2}$ photoreduction. Sci. Rep. 2014. 4. P. 6341-6347.

26. Park H., Chang S., Jean J., Jayce J., Cheng J., Araujo P.T. et al., Graphene cathode-based $\mathrm{ZnO}$ nanowire hybrid solar cells. Nano Lett. 2013. 13. P. 233-236.

27. Hasan K., Sandberg M.O., Nur O., Willander M. Transparent electrodes: $\mathrm{ZnO} /$ polyfluorene hybrid LED on an efficient hole-transport layer of graphene oxide and transparent graphene electrode. Adv. Opt. Mater. 2014. 2, No. 4. P. 304-308.

28. Pan X., Yang M.Q., Xu Y.J. Morphology control defect engineering and photoactivity tuning of $\mathrm{ZnO}$ crystals by graphene oxide - a unique 2D macromolecular surfactant. Phys. Chem. Chem. Phys. 2014. 16. P. 5589-5599.

29. Biroju R.K., Tilak N., Rajender G., Dhara S., Giri P.K. Catalyst free growth of $\mathrm{ZnO}$ nanowires on graphene and graphene oxide and its enhanced photoluminescence and photoresponse. Nanotechnol. 2015. 26. P. 601-612.

30. Szabo T., Berkesi O., Forgo P., Josepovits K., Sanakis Y., Petridis D. and Dekany I. Evolution of surface functional groups in a series of progressively oxidized graphite oxides. Chem. Mater. 2006. 18. P. 2740-2749.

31. Mkhoyan K.A., Contryman A.W., Silcox J. et al., Atomic and electronic structure of grapheme oxide. Nano Lett. 2009. 9, No 3. P. 1058-1063.

32. Young S.J., Liu Y.H., Hsiao C.H., Chang S.J., Wang B.C., Kao T.H., Tsai K.S., San-Lein W. ZnObased ultraviolet photodetectors with novel nanosheet structures. IEEE Trans. Nanotechnol. 2014. 13, No 2. P. 238-247.

33. Park C., Lee J., Sob H.M., Chang W.S. An ultrafast response grating structural $\mathrm{ZnO}$ photodetector with back-to-back Schottky barriers produced by hydro-thermal growth. J. Mater. Chem. 2015. 3. P. 2737-2746.

34. Liu H., Sun Q., Xing J., Zheng Z., Zhang Z., Lu Z., Zhao K. Fast and enhanced broadband photoresponse of a $\mathrm{ZnO}$ nanowire array/reduced graphene oxide film hybrid photodetector from the visible to the near-infrared range. ACS Appl. Mater. Interfaces. 2015. 7, No 12. P. 6645-6646.

35. Chang H., Sun Z., Ho K.F., Tao X., Yan F., Kwok W.M., Zheng Z. A highly sensitive ultraviolet sensor based on a facile in situ solution-grown $\mathrm{ZnO}$ nanorod/graphene heterostructure. Nanoscale. 2011. 3. P. 258-266.

36. Boruah B.D., Ferry D.B., Mukherjee A., Misra A. Few-layer graphene/ZnO nanowires based high performance UV photodetector. Nanotechnol. 2015. 26. P. 235-237.

37. Fu X.W., Liao Z.M., Zhou Y.B., Wu H.C., Bie Y.Q., $\quad \mathrm{Xu} \quad$ J., $\quad \mathrm{Yu}$ D.P. Graphene/ZnO nanowire/graphene vertical structure based fastresponse ultraviolet photodetector. Appl. Phys. Lett. 2012. 100. P. 223-224.

38. Khoa N.T., Kim S.W., Yoo D.H., Cho S., Kim E.J., Hahn S.H. Fabrication of Au/graphene-wrapped $\mathrm{ZnO}$-nanoparticle-assembled hollow spheres with effective photo-induced charge transfer for photocatalysis. ACS Appl. Mater. Interf. 2015. 7, No. 6. P. 3524-3528.

39. Lin C.L., Chang W.Y., Huang Y.L. et al. J. Appl. Phys. 2015. 54. P. 4-8.

40. Fouda A.N., El Basaty A.B. and Eid E.A. Photoresponse of functionalized self-assembled graphene oxide on zinc oxide heterostructure to UV illumination. Nanoscale Res. Lett. 2016. 1. P. 1-8.

41. Ab initio calculation [E-resource] - Mode access to the resource: http://sites.google.com/a/kdpu.edu.ua/ calculationphysics. 
42. Topsakal M., Cahangirov S., Bekaroglu E., and Ciraci S. First-principles study of zinc oxide honeycomb structures. Phys. Rev. B. 2009. 80. P. 235119.

43. Tusche C., Meyerheim H.L. and Kirschner J. Observation of depolarized $\mathrm{ZnO}(0001)$ monolayers: Formation of unreconstructed planar sheets. Phys. Rev. Lett. 2007. 99. P. 026102.

\section{Authors and CV}

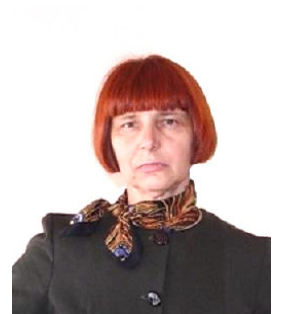

Ruslana Balabai received her Ph.D. degree from South Ukrainian National Pedagogical University named after K. D. Ushynsky in 1993. She received her degree of Doctor of Physical and Mathematical Sciences in the Department of Physics and Teaching Methodology in the Kryvyi Rih State Pedagogical University in 2014. Her research focuses on $a b$ initio calculations in condensed matter, novel electronic/optoelectronic devices based on graphene, other 2D nano-materials. Now Ruslana Balabai is professor in the Department of Physics and Teaching Methodology.

Kryvyi Rih State Pedagogical University

E-mail:balabai@i.ua

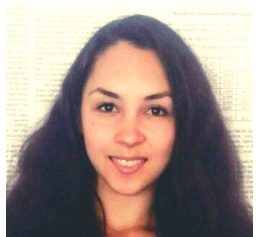

Zdeshchyts Anastasiia graduated from the Kryvyi Rih State Pedagogical University in 2015. Today she is a post-graduate student at the Department of Physics and Teaching Methodology in the Kryvyi Rih State Pedagogical University. Her research focuses on $a b$ initio calculations in condensed matter, hybrid nanostructures, «green» electronics, other 2D nano-materials. Now Anastasiia Zdeshchyts is a junior researcher at the Research Mining Institute.

Kryvyi Rih State Pedagogical University

E-mail: bodehita@gmail.com

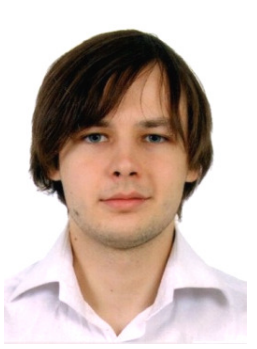

D.V. Zalevskyi received his master's degree in the Kryvyi Rih State Pedagogical University in 2018. His research focuses on $a b$ initio calculations of electronic properties of 2D nano-materials.

Kryvyi Rih State Pedagogical University

E-mail: zalev.denisk@mail.ru 